\title{
Einsatzmöglichkeiten kleiner Fixateure
}

\author{
Alexander Möbius, Dara Orangi, Karl Heinrich Winker
}

\section{Zusammenfassung}

Neben den bekannten Techniken der internen Osteosynthese zur Frakturstabilisierung an der Hand hat sich die Verwendung eines kleinen und/ oder des Mini-Fixateur externe einen festen Platz im handchirurgischen Repertoire erobert. Die Vorteile liegen in der Möglichkeit einer indirekten Frakturreposition und der daraus resultierenden minimalinvasiven Vorgehensweise. Bei offenen Verletzungen mit schwierigen Weichteilverhältnissen sowie bei Infektionen ist der Fixateur die Methode der Wahl. Die technische Auslegung des Mini-Fixateurs wurde mittlerweile den Dimensionen des Handskeletts angepasst. Neben der Beachtung allgemeiner Richtlinien für die Anwendung des Fixateur externe muss ein hohes Maß an funktionellem Verständnis der Bewegungsabläufe der Hand realisiert werden. Bei entspre- chender Konstruktion soll bei den meisten Patienten eine Überbrückung frakturangrenzender Gelenke vermieden werden. Durch die konsequente postoperative Beübung der Gelenke wird eine höhergradige Einsteifung verhindert. Als Nachteil der Fixateurbehandlung wird die notwendige, regelmäßige Pflege der Fixateur-Pins empfunden. Bei entsprechender Erfahrung treten die Pin-Infektionen selten auf. So bleibt der Fixateur als ideale temporäre Stabilisierung und auch als definitive Versorgung im Einsatz.

\section{Potential Uses of the Small Fixator}

In addition to the well known techniques of internal fixation, the use of a small and/or minifixator in fracture stabilisation of the hand has gained in popularity among hand surgeons. The advantage of this technique is the possibility for a closed reduction resulting in a minimally invasive operative technique. The minifixator is the first choice of treatment in open or infected cases of fractures and also in cases with complicated soft tissue lacerations. The technical features of the fixator must take into account the dimension of the hand skeleton. A knowledge of the basics of fixator use for fracture treatment must be accompanied by a detailed knowledge about anatomic and functional hand physiology. With an adequate apparatus in most of the cases, a joint-bridging application of the fixator can be avoided. A consequent postoperative physiotherapy should start early to reduce a risk of stiffness. A disadvantage is the necessity for regular pin care. However, with adequate experience it can be reduced to a minimum. The fixator is a very useful apparatus for temporary stabilisation and also as a standard surgical fracture treatment procedure.

\section{Einleitung}

Die menschliche Hand ist wegen der besonders hohen Exposition im Alltags- und Berufsleben dem Verletzungsrisiko ausgesetzt. Dementsprechend oft kommt es zu Frakturen des Handskeletts. Die Behandlung soll den zu erwartenden funktionellen Erfordernissen gerecht werden.

Neben den allgemeinen Gesichtspunkten der zuverlässigen Wiederherstellung der Skelettanatomie muss an der Hand die besondere Aufmerksamkeit den Weichteilen geschenkt werden.
OP-JOURNAL 2008; 24: 38-43

(c) Georg Thieme Verlag KG Stuttgart • New York DOI 10.1055/s-2008-1038436
Das empfindliche Sehnengleitgewebe darf weder durch den chirurgischen Eingriff (ausgedehnte Zugänge) noch durch prolongierte, postoperative Immobilisation der Gelenke geschädigt werden. Auch die Kontrakturen der Gelenkkapsel und des Bandapparats lassen sich ergotherapeutisch nur schwer behandeln.

Die Indikationsstellung muss grundsätzlich von der Forderung ausgehen, eine möglichst frühe Mobilisation der angrenzenden Gelenke zu ermöglichen, ohne Reposition und Frakturheilung zu gefährden.

Eine nach wie vor gültige klassische Indikation zur Fixateur-externe-Behandlung besteht bei gelenknahen Trümmerfrakturen nach dem Prinzip der Ligamentotaxis. Durch den Zug der Weichteile kommt es auch bei zunächst hoffnungslos erscheinenden Trümmerfrak- turen zu erstaunlich guten Repositionsergebnissen. Eine weitere klassische Indikation beinhaltet die Behandlung der offenen Frakturen und die Osteomyelitistherapie. Die externe Stabilisierung mit einem geeigneten kleinen oder MiniFixateur verbindet die Vorteile der sicheren Stabilisierung mit einem minimalinvasiven Eingriff (Tab.1).

\section{Anatomische Vorbemerkungen}

Die externe Stabilisierung im Handgelenk und Mittelhandbereich erfolgt von dorsal aus.

Im Bereich der Mittelhand bereitet die Platzierung von Schanz-Schrauben oder Pins erfahrungsgemäß keine Schwierigkeiten, jedoch aufgrund der typischen Strecksehnenkonvergenz zum Handgelenk hin sind im proximalen Mittelhandbereich die sicheren Applikations- 
Tab. 1 Indikationen zur Anlage eines Fixateur externe im Hand- und Fußbereich

\begin{tabular}{l} 
- gelenknahe Trümmerfrakturen \\
\hline - offene Frakturen \\
\hline - Defektfrakturen \\
\hline - Luxationen und Luxationsfrakturen der Handwurzel \\
\hline - Frakturen mit schwerem geschlossenen Weichteilschaden \\
\hline - komplexe Weichteilverletzungen ohne Frakturen \\
\hline - Mehrfachverletzte \\
\hline - Infektionen (infizierte Frakturen)
\end{tabular}

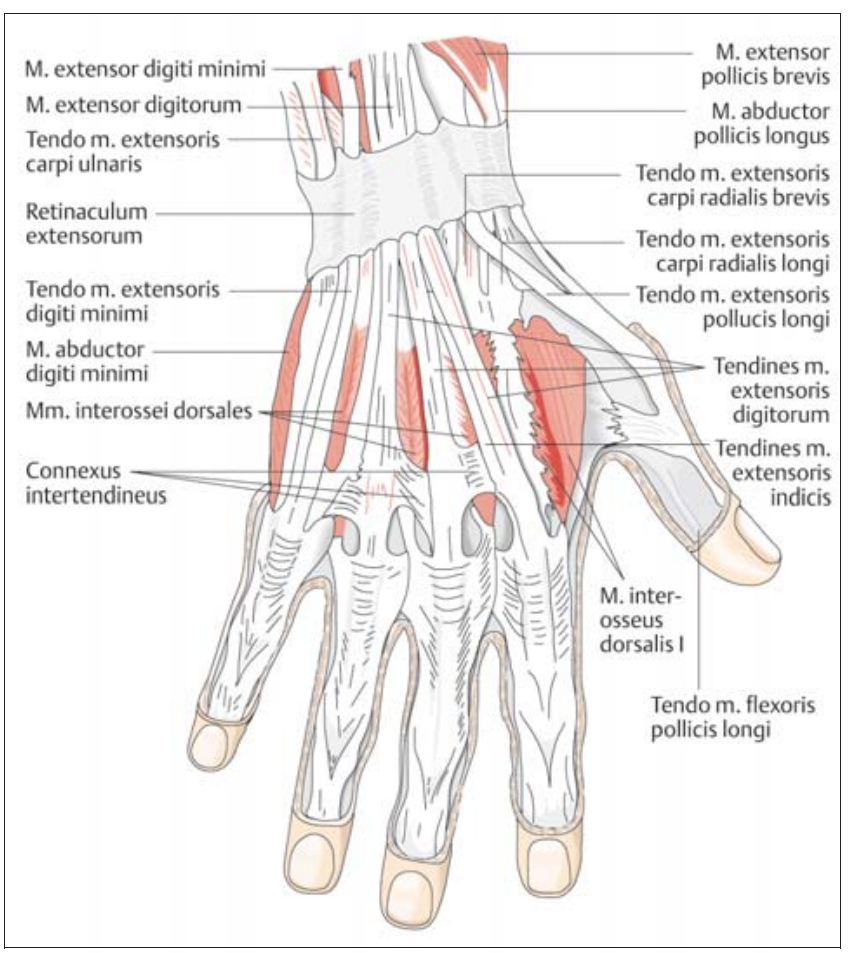

korridore ausgesprochen klein, sodass hier zur sicheren Vermeidung einer Strecksehnentransfixierung eine kleine Stichinzision notwendig ist.

Die randständigen Strahlen II und V sind wesentlich leichter erreichbar als die Zentralstrahlen III und IV.

Müssen aufgrund der Frakturlokalisation Fixateurschrauben oder Pins in der Nähe des Mittelhandknochenköpfchens eingebracht werden, so ist der typische Verlauf des Kollateralbandes zu beachten. Die perkutane Applikation von Fixateur-Pins im Bereich des Fingergrundgliedes ist technisch anspruchsvoller, da vor allem der proximale Schaft von der Streckerhaube vollständig überlagert ist. Das Durchbohren der Streckerhaube in ihrem mittleren Anteil führt jedoch
Abb. 1 Strecksehnenanatomie Hand und Finger.

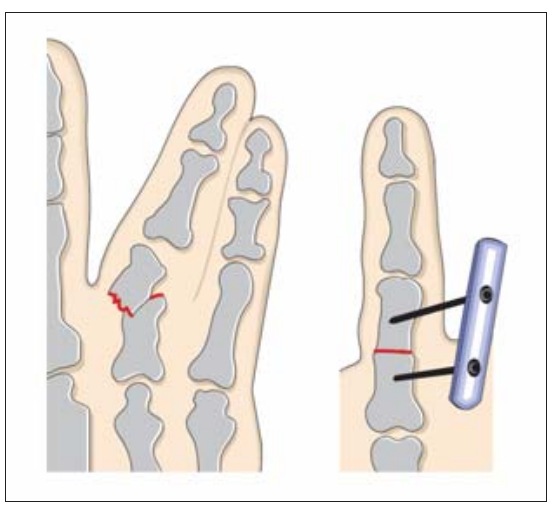

Abb. 2 Schematisierte Nachbildung des Lambotte-Fixateurs, 1904.

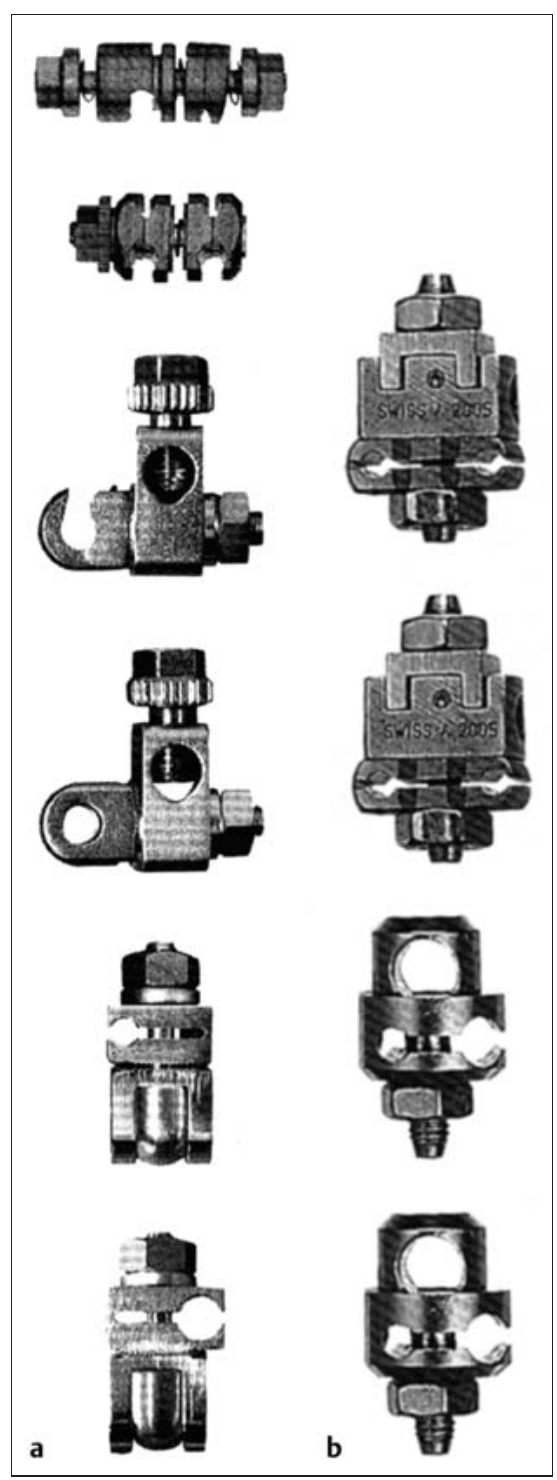
sitionieren wir die Schanz-Schrauben im Winkel von $20-30^{\circ}$ zur Horizontalebene (dorso-radial), um beispielsweise bei distalen Radiusfrakturen die problemlose Lagerung des Unterarmes für die spätere Versorgung durch palmare Plattenosteosynthese zu ermöglichen. 


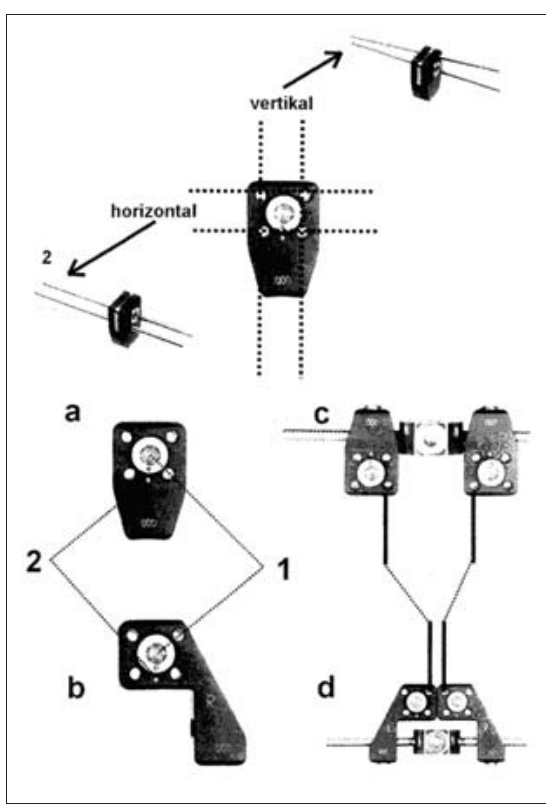

Abb. 4 Mini-Fixateur der Fa. Sulzer Medica, Orthofix Srl., Italien, 1994.
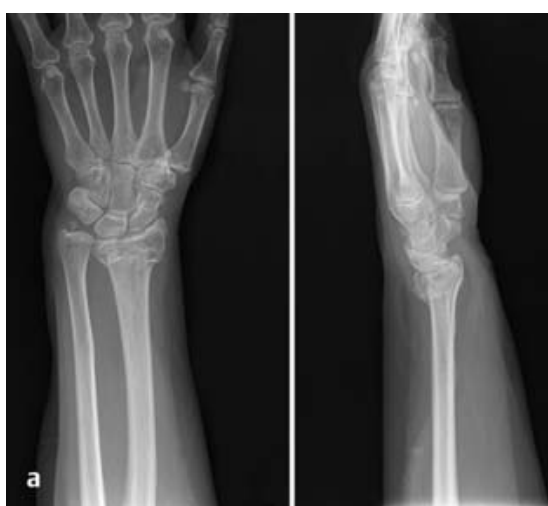

Abb.5a Röntgenbild einer intraartikulären distalen Radiusfraktur.

\section{Das Fixateursystem}

Bereits im Jahr 1904 behandelte Lambotte eine Fingergrundgliedfraktur mit einem selbstkonstruierten Fixateur externe (Abb. 2). Er verwendete je eine Fixateurschraube diesseits und jenseits des Frakturspaltes, die mit einer starren Stange verbunden waren.

Die modernen Systeme erlauben nach der Platzierung der Pins/Schanz-Schrauben bevorzugt paarweise in den Hauptfragmenten über die Anwendung der Modulartechnik eine Feinreposition über eine Vielzahl der Backen für Verbindungen Stäbe/Pins oder Stäbe/ Schanz-Schrauben (Abb. 3).

Die Möglichkeit zur Verankerung von Fixateur-Pins in kleinen, periartikulären Fragmenten sowie die Möglichkeit zur

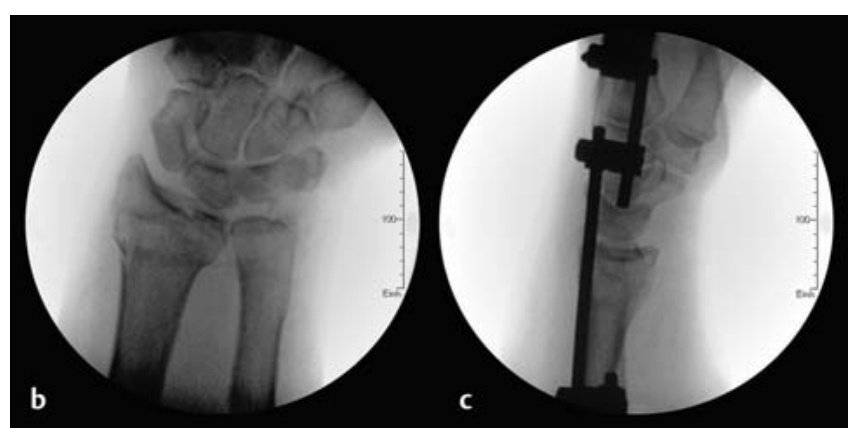

Abb. 5 b und $c$

Reposition im Fixateur externe durch Ligamentotaxis.
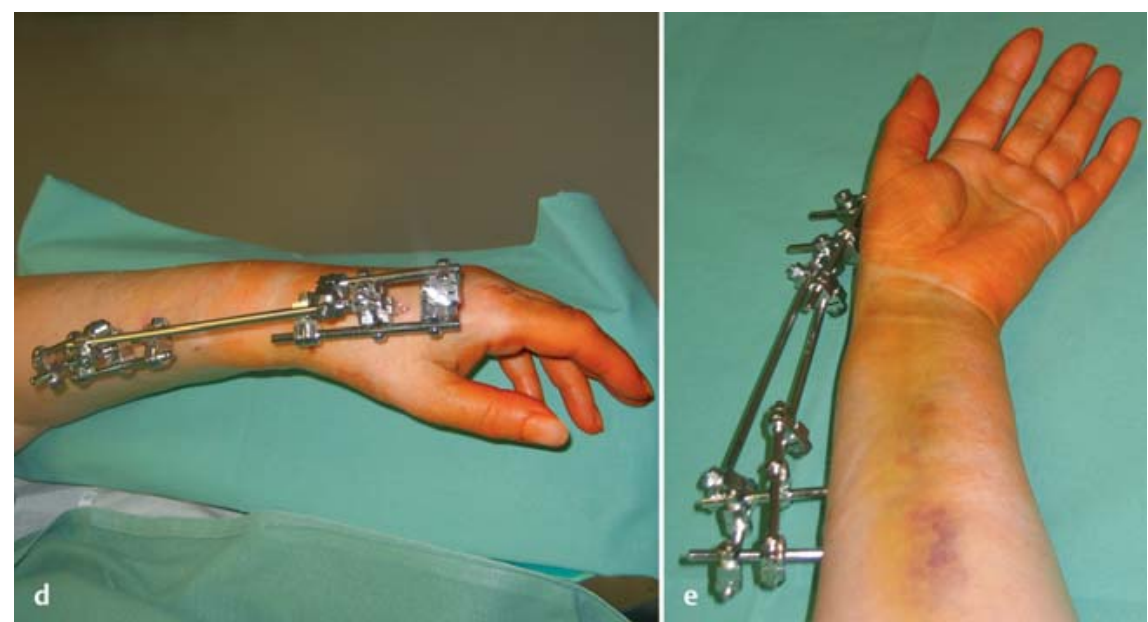

Abb. 5d und e Klinisches Bild mit problemloser Lagerung auf dem OP-Handtisch zur Durchführung einer palmaren Plattenosteosynthese bei liegendem Fixateur externe.
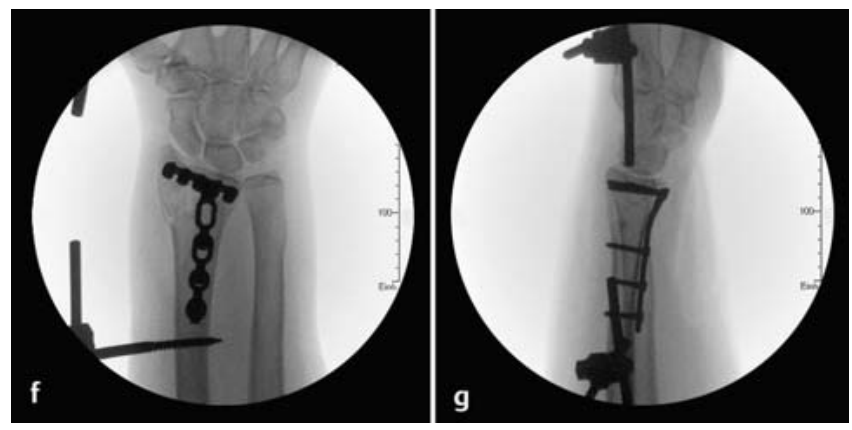

Abb. $5 f$ und $g$ Nach Optimierung der Weichteile erfolgte die palmare winkelstabile Plattenosteosynthese.

Feinreposition der Fraktur sind an der Hand von größerer Bedeutung als die Erzeugung hoher Rigidität.

Eine Reihe von Mini-Fixateuren steht zur Verfügung. Es handelt sich im Wesentlichen um miniaturisierte Versionen der am langen Röhrenknochen angewendeten Apparate (Abb.4).

\section{Fixateur externe im Handgelenks- bereich}

Die distale Radiusfraktur ist einer der häufigsten Knochenbrüche am menschlichen Skelett.
Bei der Behandlung der kompliziertesten Frakturen (AO Typ 23-C2/C3) sowie bei den offenen Frakturen wird die Anwendung des Fixateur externe als Mittel der Wahl gesehen.

\section{Operationstechnik}

Der Fixateur wird unilateral angebracht. Die Operation wird unter aseptischen Bedingungen im OP-Saal entweder in Plexusanästhesie oder in Intubationsnarkose durchgeführt. Blutsperre in Bereitschaft. Wir verwenden das AO-Instrumentarium (kleiner Fixateur externe der Firma Synthes). 


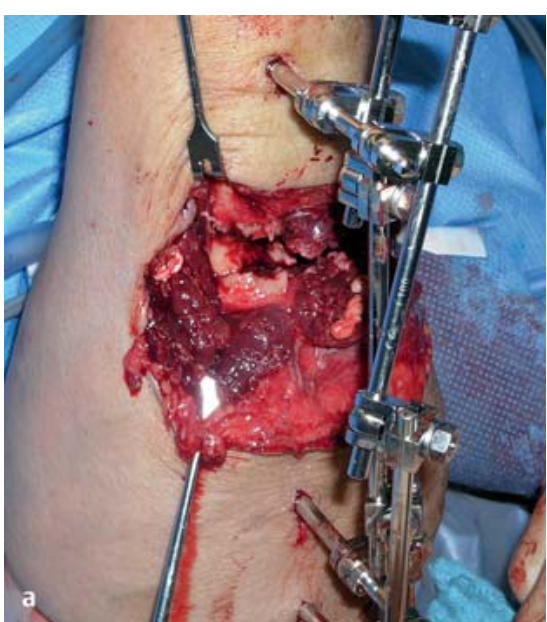

Abb.6a Kettensägenverletzung des Unterarms mit $2^{\circ}$ offener Defektfraktur der Ulna.
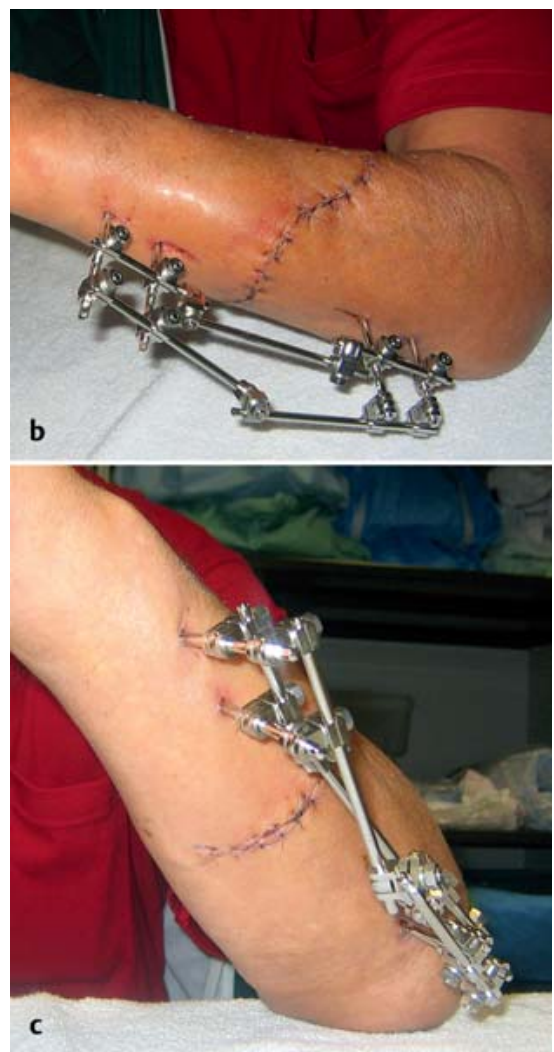

Abb.6b und c Primäre Wundversorgung und Stabilisierung der Ulna mit kleinem Fixateur externe.

Grundsätzlich erfolgt nach Hautinzision über dem Metakarpale II und dem distalen Radius die stumpfe Dissektion des Subkutangewebes bis auf den Knochen, um Läsionen von Sehnen und Nerven zu vermeiden.

Es werden die 2,7-mm- bzw. 3,5-mmSchanz-Schrauben mit einem Schaftdurchmesser von $4 \mathrm{~mm}$ eingebracht.
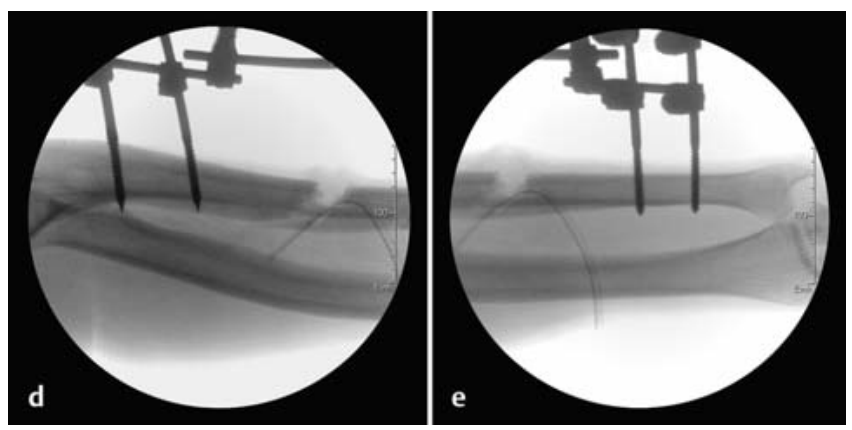

Abb. $6 \mathrm{~d}$ und $\mathrm{e}$ Röntgenologisch gesicherte anatomische Stellung der Ulna.

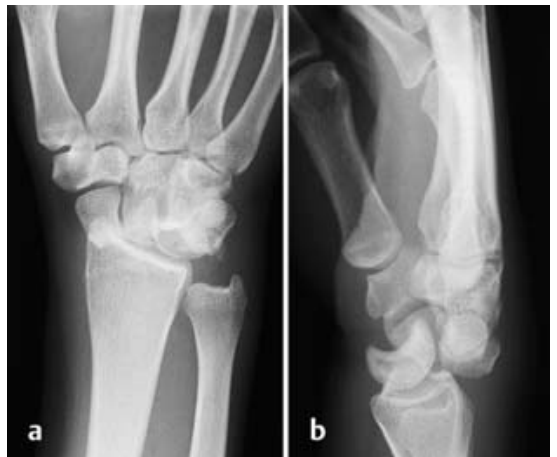

Abb. 7a und b DeQuervain'sche perilunäre transskaphoidale Luxationsfraktur.

Das Auffinden der Bohrstelle wird durch den temporär eingebrachten 2,0-mmK-Draht erheblich erleichtert. Die Positionierung erfolgt relativ flach zur Horizontalebene $\left(\max .30^{\circ}\right.$ ), um die spätere definitive Versorgung vorzugsweise durch palmare winkelstabile Plattenosteosynthese oder simultane zusätzliche perkutane Schraubenosteosynthese durch ungehinderte Unterarmlagerung auf dem Handtisch zu ermöglichen. Die Stangenmontage erfolgt modular, wobei die kurzen Stangen jeweils parallel zur Schaftachse des Metakarpale II und des distalen Radius fest montiert werden und danach durch eine Mittelstange nach Reposition der Fraktur miteinander verbunden werden. Nach Durchleuchtungskontrolle bei gutem Repositionsergebnis erfolgt die Erhöhung der Stabilität des Fixateurs durch eine zweite parallele Stangenmontage (Abb. 5 a bis e).

Postoperativ wird bei intraartikulären Frakturen durch eine CT-Diagnostik die Möglichkeit einer internen Osteosynthese überprüft (Abb. $\mathbf{5} \mathbf{f}$ und $\mathbf{g}$ ).

Die definitive Fixateur-externe-Versorgung von distalen Radiusfrakturen bedarf regelmäßiger Röntgenkontrollen der Frakturstellung (2., 4. und 6. Woche). Nach ca. 2 Wochen wird bei primär

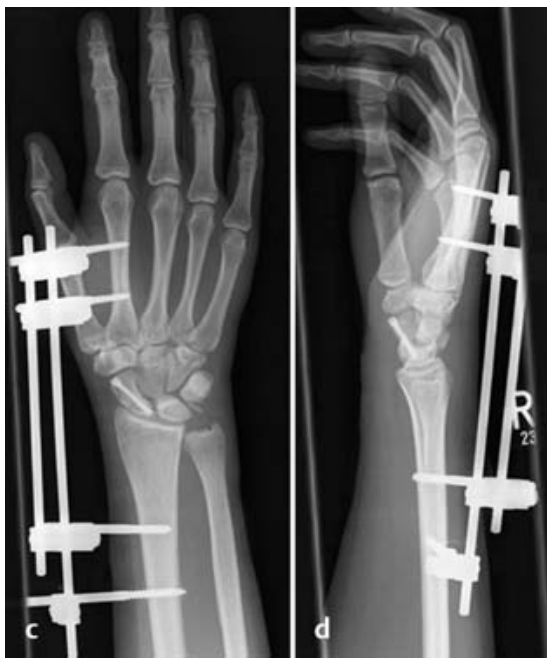

Abb. 7c und d Repositionsergebnis nach offener Schraubenosteosynthese des Skaphoids und Fixateur-externe-Anlage.

in Extension eingestellten Frakturen der Zug nachgelassen, um die Kontraktur der periartikulären Weichteilstrukturen zu verhindern. Am Ende der 6. postoperativen Woche wird in der Regel ohne Betäubung die Metallentfernung vorgenommen. Bewegungsübungen der Finger beginnen unmittelbar postoperativ.

\section{Defektfrakturen}

Bedingt durch den Schweregrad der Weichteilverletzung und Kontamination verbietet sich bei direkten Gewalteinwirkungen wie z.B. Kettensägenverletzungen eine primäre interne Osteosynthese (Abb. 6), der kleine Fixateur externe ist auch hier ein ideales Fixationsinstrument.

\section{Karpusluxationen und Luxationsfrakturen}

Es reißen dabei entweder die dorsalen oder die palmaren Bänder nicht selten kombiniert mit Frakturen des Radius oder randständiger Handwurzelknochen der proximalen Reihe. Reine kar- 


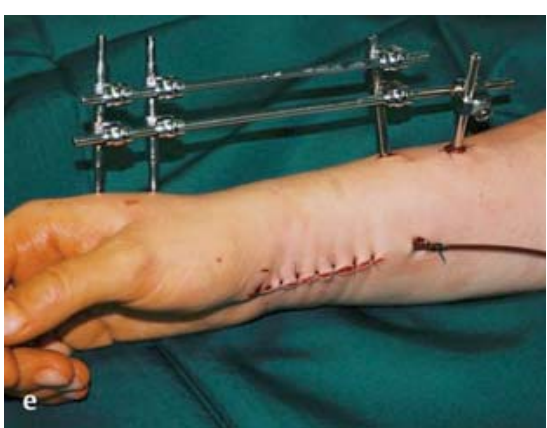

Abb.7e Klinisches Bild mit sofortigem Beginn der Fingerübungen.

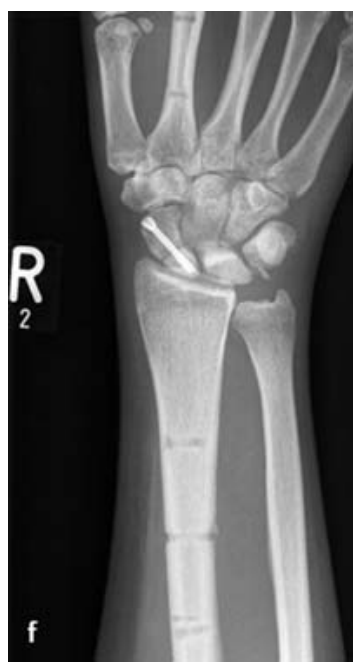

Abb. 7f

Nach 6 Wochen Entfernung des Fixateurs, Intensivierung der Ergotherapie.

pale Luxationen heilen nach korrekter Reposition und Retention im Fixateur externe in der Regel gut aus. Die Luxationsfrakturen bedürfen einer zusätzlichen perkutanen oder offenen Schraubenosteosynthese der jeweiligen Frak$\operatorname{tur}$ (Abb. 7).

\section{Frakturen der Mittelhand und Finger}

Es gelten die üblichen Kriterien der Osteosynthese. Instabile Trümmerfrakturen im Bereich der Hand lassen sich durch Mini-Fixateur gut überbrücken. Grundsätzlich sollen aber hier die Fixateur-Pins durch offenes Vorgehen platziert werden. Zum Beispiel die radiale Seite des Metakarpale I und Trapezius bei der Versorgung einer WintersteinFraktur wird vom dorsalen Ast der A. radialis und vom Ramus superficialis $n$. radialis überlagert (Abb. 8).

Die Stabilität des Mini-Fixateurs soll eine sofortige postoperative Beübung zulassen. Zum Abschluss der Operation wird die freie Beweglichkeit der angrenzenden Fingergelenke überprüft und so

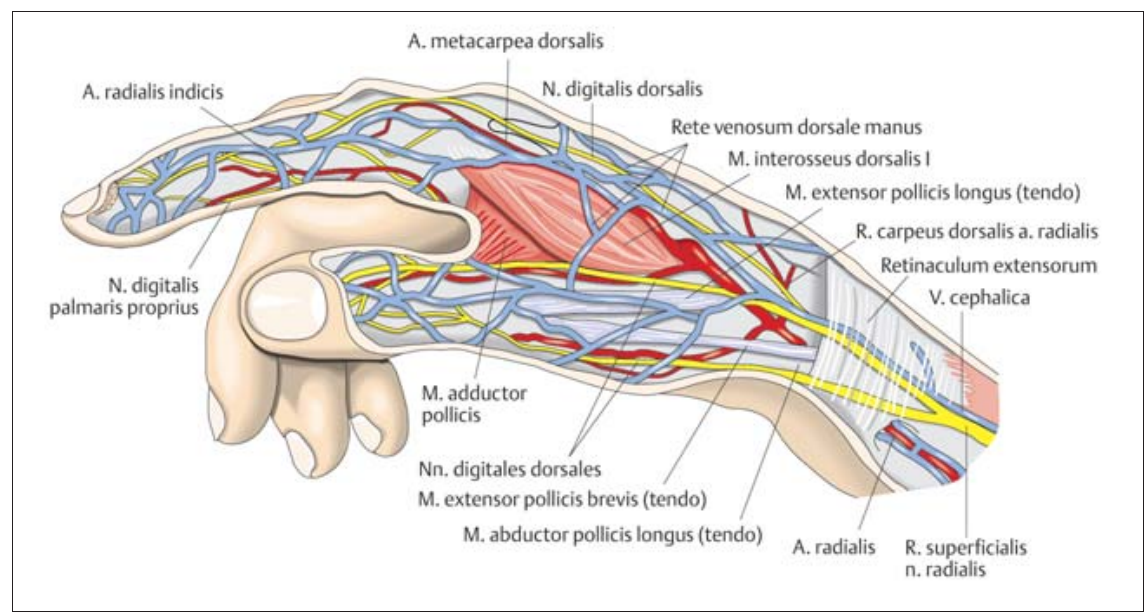

Abb. 8 Anatomische Dichte der Gefäß-Nerven-Strukturen im seitlichen Handaspekt.

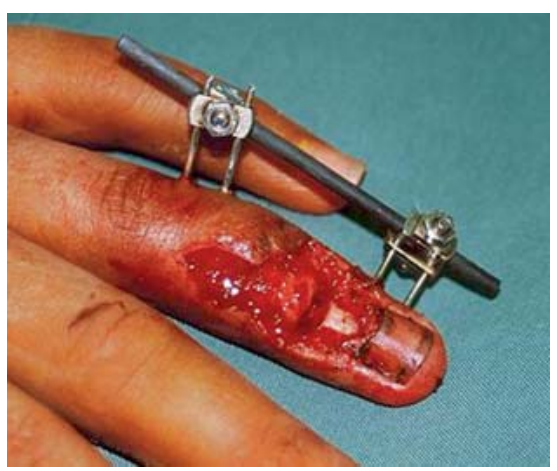

Abb. 9 Offene Defektverletzung im Bereich des DIP-Gelenks. Anlage des Mini-Fixateurs vor Weichteildeckung.
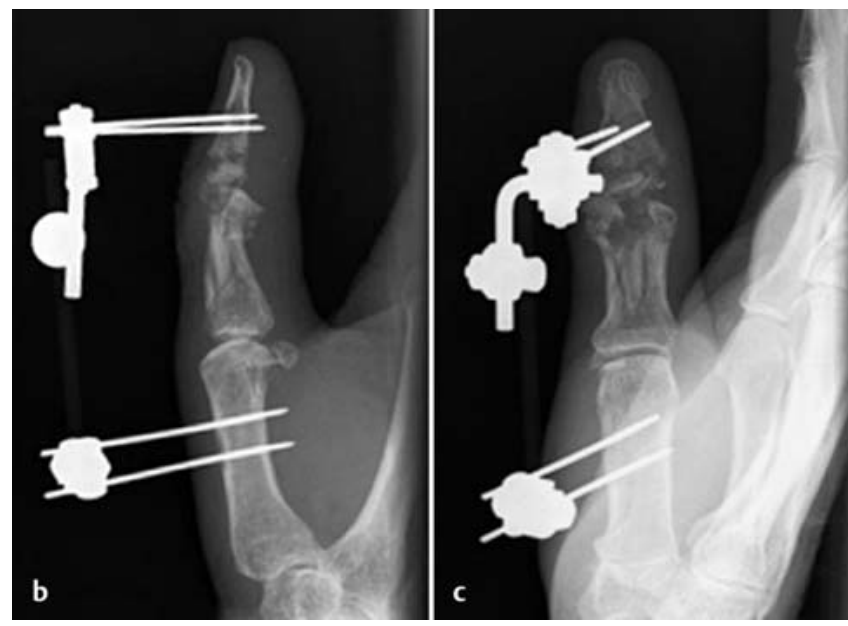

die Übungsstabilität der Montage dokumentiert.

Die Technik der externen Fixation an den Phalangen ist sehr anspruchsvoll und soll dem Erfahrenen vorbehalten bleiben.

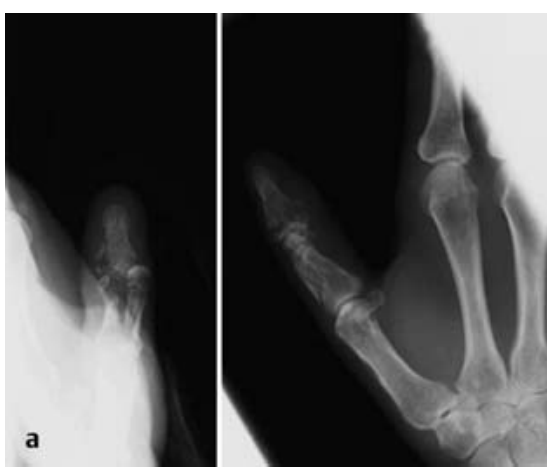

Abb. 10a Röntgenbilder einer Berstungsfraktur des Grund- und Endglieds des Daumens. Zerstörung des IP-Gelenks.

Abb. $10 \mathrm{~b}$ und $\mathrm{c}$ Röntgenbilder nach Reposition durch Ligamentotaxis im Mini-Fixateur. 


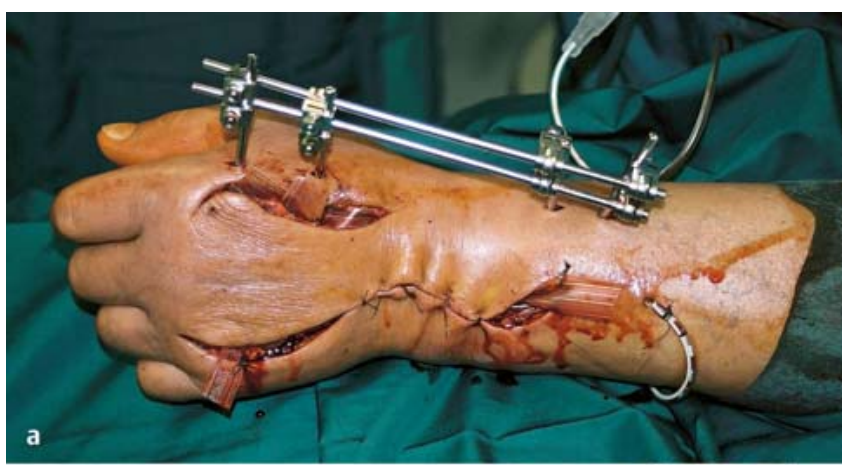

Abb. $11 \mathrm{a}$ und $\mathrm{b}$ Handgelenksempyem nach palmaren und dorsalen Débridements, Anlage einer Spül-Saug-Drainage, Stabilisierung des Gelenks mit Fixateur externe.

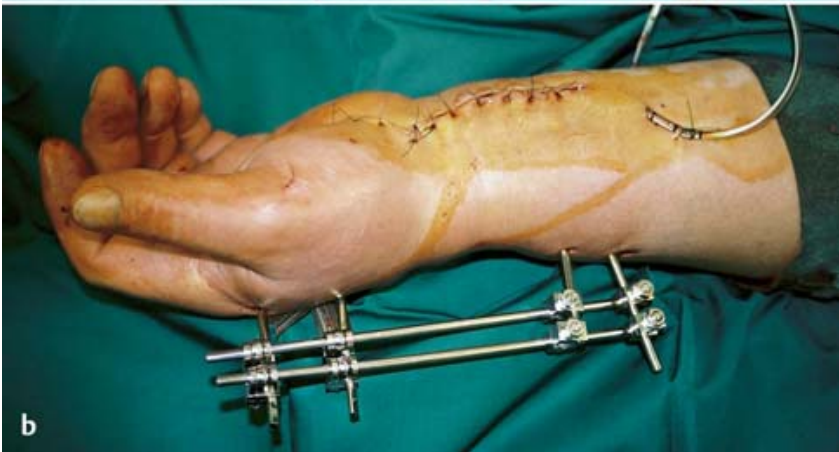

\section{Infektionen}

Die zuverlässige Stabilisierung und minimalinvasive Applikationstechnik macht den Fixateur zum Mittel der Wahl bei Infektionen im Handgelenk und Handbereich (Abb. 11).

\section{Komplikationen und Probleme der Fixateur-externe-Behandlung}

Bei distalen Radius-Gelenkfrakturen (23-C) können nach geschlossener Reposition und Anlage des kleinen Fixateurs Stufenbildungen in der distalen Radiusgelenkfläche und eine Inkongruenz im distalen Radioulnargelenk (DRUG) verbleiben. Dies kann zu einer posttraumatischen Arthrose und/oder Funktionseinschränkungen vor allem bei der Unterarmdrehbewegung führen.

Durch starke Distraktion während der Ruhigstellung mit Fixateur externe besteht die Gefahr einer Reflexdystrophie oder Kontraktur der periartikulären Weichteile (s.o.).

Infekte im Bereich der Schanz-Schrauben können meist durch geübte Pin-
Pflege und auch ohne Verlust der Reposition durch Umsetzen der Pins beherrscht werden.

Die mögliche Lockerung der SchanzSchrauben vor allem beim osteoporotischen Knochen limitiert sicher die definitive Anwendung des Fixateur externe über mehrere Wochen hinweg bis zur knöchernen Konsolidierung.

\section{Schlussfolgerung}

Die Anwendung des kleinen oder MiniFixateur externe an Unterarm und Hand wie in ausgewählten Fällen auch am Fuß ist zu einer unverzichtbaren Operationsmethode bei bestimmten Indikationen geworden.

Falls technisch durchführbar soll eine gelenküberbrückende Montage vermieden werden. Unter Beachtung der anatomischen Gegebenheiten lässt sich eine Sehnentransfixation oder Verletzung der Gefäß-Nerven-Bündel vermeiden. Das Barton'sche Axiom ,the best way to make the hand work is to make the hand work" lässt sich auch mit diesem Verfahren erreichen [4].

\section{Literatur}

1 Asche G. Stabilisierungsmöglichkeit einer intraartikulären Trümmerfraktur des ersten Mittelhandkochens mit dem Minifixateur externe. Handchirurgie 1981; 13: 247 - 249

2 Asche G, Burny F. Indikation für die Anwendung des Minifixateur externe. Eine statistische Analyse. Akt Traumatol 1982; 12: $103-$ 110

${ }^{3}$ Ashmead D, Rothkopf DM, Walton RI, Jupiter JB.Treatment of hand injuries by external fixation. J Hand Surg [Am] 1992; 17: 956 - 964

${ }^{4}$ Barton NJ. Fractures of the hand. J Bone Joint Surg [Br] 1984; 66: 159-167

${ }^{5}$ Gausepohl T, Koebke J, Pennig D, Hobrecker S. Anatomische Grundlagen zur Anwendung der unilateral externen Fixation an Oberarm, Unterarm und Hand. Osteosynthese Intern 1997; 5: 76-88

${ }^{6}$ Gausepohl T, Lukosch S, Koebke J, Pennig D. Externe Stabilisierung der Mittelhandknochen II bis V. Eine anatomisch-klinische Studie. Handchir Mikrochir Plast Chir 1998; 30: 95-102

${ }^{7}$ Green DP, O'Brien ET. Fractures of the thumb metacarpal. South Med J 1972; 65: 807-814

${ }^{8}$ Hastings H. Unstable metacarpal and phalangeal fracture treatment with screws and plates. Clin Orthop 1987; 214: 37-52

9 Parsons SW, Fitzgerald JAW, Shearer JR. External fixation of unstable metacarpal and phalangeal fractures. J Hand Surg [Br] 1992; 17: $151-155$

${ }^{10}$ Pellegrini VD. Fractures of the base of the thumb. Hand Clin 1988; 4: 87-101

11 Winker KH, Schmidt J. Der Stellenwert des externen Fixateurs bei der Behandlung der distalen Radiusfraktur. Stuttgart: Thieme, 1999

\section{Alexander Möbius \\ Oberarzt \\ Dr. med. Dara Orangi \\ Leitender Oberarzt \\ Prof. Dr. med. Karl Heinrich Winker Chefarzt}

Klinik für Unfall-, Hand- und Wiederherstellungschirurgie Helios Klinikum Erfurt Nordhäuser Straße 74 99089 Erfurt 